\title{
La comunicación de la banca hacia sus clientes: evolución y el modelo alternativo de la banca islámica
}

\author{
$M^{\mathrm{a}}$ del Carmen de la ORden DE la CRUZ \\ Universidad Rey Juan Carlos de Madrid (España) \\ carmen.delaorden@urjc.es \\ Graciela Padilla CAStillo \\ Universidad Complutense de Madrid (España) \\ gracielapadilla@ccinf.ucm.es \\ Pilar SÁNCHEZ GoNZÁLEZ \\ ESIC Business \& Marketing School de Madrid (España) \\ pilar.sanchez@esic.edu
}

\begin{abstract}
Resumen
La reciente crisis ha puesto de manifiesto los problemas de la comunicación de la banca respecto a sus clientes, agudizados por la escasa formación financiera de los ciudadanos. Así, se hace evidente la necesidad de un cambio de la estrategia comunicativa en este sector y la búsqueda de nuevos métodos y enfoques. En este trabajo tratamos de analizar los cambios acaecidos en los últimos años en cuanto a comunicación bancaria y cómo la banca islámica se constituye como referente en la búsqueda de un modelo alternativo.
\end{abstract}

Palabras clave: Banca Islámica, comunicación al cliente bancario, estrategia comunicativa, personalización, profesionalización

Communication to its customers banking: evolution and the alternative model of Islamic banking

\begin{abstract}
The recent crisis has highlighted the problems of banking communication regarding their customers, exacerbated by the lack of financial education of citizens. Thus, it becomes evident the need for change communication strategy in this sector and the search for new methods and approaches. In this work we analyze the changes in recent years in terms of banking communication and how the Islamic banking could be a reference.
\end{abstract}

Key Words: Islamic banking, banking customer communication, communication strategy, personalization, professionalism

\section{Referencia normalizada:}

De la Orden de la Cruz, M. C.; Padilla Castillo, G. y Sánchez González, P. (2013) La comunicación de la banca hacia sus clientes: evolución y el modelo alternativo de la banca islámica. Historia y Comunicación Social. Vol. 18. № Especial Diciembre. Págs. 575-585.

Sumario: 1. Introducción. 2. Marco metodológico. 3. Desarrollo. 4. Conclusiones. 5. Bibliografía. 


\section{Introducción}

La crisis de los últimos años ha tenido un coste en todos los ámbitos, pero también ha supuesto un elemento dinamizador de nuevas formas de trabajar en finanzas. En el caso del mundo financiero y en concreto, en el ámbito de la comunicación con respecto al cliente, se han evidenciado sus debilidades. La escasa formación bancaria de los clientes junto con la insuficiente y, no siempre clara, información que se da a éste, han empeorado la imagen del sector que, tradicionalmente, se ha percibido como negativa. De este modo, nos encontramos ante un cambio necesario de estrategia comunicativa y, por tanto, una exploración de nuevos métodos y enfoques.

Ni el mismo tipo de mensajes, ni el mercado, ni los clientes son los mismos que hace unos años. Ahora, bajo un entorno de crisis y un mercado global, los clientes necesitan poder confiar, más que nunca, en sus entidades financieras. No basta con que, simplemente, se comercialicen servicios financieros, sino que se demanda un asesoramiento con comportamiento más ético, una mayor profesionalización y, sobre todo, un trato personalizado.

Nuestro trabajo trata de analizar los cambios acaecidos en los últimos años en cuanto a comunicación bancaria y cómo estos están provocando una realidad del consumidor financiero diferente que, bajo nuestro criterio, pasa por la "personalización" de las acciones. Cada entidad deberá decidir a quién se dirige, cómo y cuándo, respetando las condiciones que demanda el cliente en la prestación de un servicio financiero. En este sentido, la Comunicación en la relación banco-cliente debería ser personalizada y las entidades financieras tendrían que pormenorizar mucho más, pues ya no es suficiente con considerar variables como sexo, edad o estilos de vida. Un elemento es la religión, variable que puede considerarse muy importante desde la perspectiva estratégica de la comunicación pues los clientes, cada vez más, deciden dónde comprar e invertir según consideraciones emocionalmente éticas que están entroncadas con su religión. En nuestro caso, consideramos el Islam y la Banca Islámica como caso de estudio, planteándolos como alternativa a la banca tradicional. El objetivo es demostrar la evolución que ha tenido el sector financiero desde la comunicación masiva hacia la personalización, de forma generalizada, y religiosa, de forma muy concreta.

\section{Marco metodológico}

Hemos empleado una revisión bibliográfica, en versión analítica-cualitativa, para cumplir los objetivos propuestos, acercarnos a la nueva comunicación financiera con el cliente, y saber cómo funcionan, en concreto, los mercados hacia las minorías. El punto de partida fue Dialnet, la base de datos de la Universidad de La Rioja, que reúne documentos en abierto de 58 bibliotecas universitarias, 4 bibliotecas públicas y 15 bibliotecas especializadas. En la búsqueda "comunicación financiera" se 
hallan 132 documentos, entre tesis, libros y artículos científicos. Estos forman parte de disciplinas muy diversas y podemos afirmar que las investigaciones, en muchos casos, son interdisciplinares pues se abordan desde estudios económicos, financieros, comunicacionales o filológicos.

Sin embargo, las palabras "banca islámica" sólo arrojan 11 resultados. Siendo Dialnet una base tan fiable y democrática, la ausencia de investigaciones de este tipo entre sus documentos es representativa del vacío bibliográfico y académico generalizado sobre el asunto. No obstante, hemos estudiado en profundidad todos los documentos propuestos por esta Web, además de otros documentos encontrados en nuestra carrera investigadora, que no trataban el asunto como elemento principal del título pero sí lo hacían en sus textos. Por ello, al mismo tiempo, este texto puede ser una prospección académica para futuras investigaciones que continúen las líneas propuestas e intenten rellenar el vacío académico sobre el asunto.

Además de Dialnet, hemos buscado investigaciones especializadas, otras publicaciones sobre comunicación financiera, diferentes teorías, escritos de autores que traten la comunicación de la banca y el marketing sobre minorías. Esos textos son fuentes primarias especializadas: artículos, libros, legislación y estadísticas. Posteriormente, se ha analizado de forma exhaustiva toda la documentación, intentando unir enfoques que cubriesen todos los aspectos reseñables y dignos de mención. Sotelo (2012: 220) expone así las virtudes de la revisión bibliográfica y documental: "Constituye uno de los principales pilares en los que se sustenta toda investigación”.

El mismo autor argumenta que dicha revisión facilita la elaboración del marco teórico y permite delimitar con mayor precisión el objeto de estudio y constatar el estado de la cuestión (Sotelo, 2012: 220). Por otro lado, Charlot (1994: 1) afirma que "una buena metodología es una metodología que permite recoger, de manera rigurosa, datos pertinentes para responder a un conjunto de preguntas". En este caso, todas nuestras preguntas se referían a conocer en profundidad cómo es la comunicación financiera a los clientes; qué importancia tienen la ética, la profesionalización y la personalización en esta comunicación; y cómo la Banca Islámica puede ser un buen ejemplo de banca minorista.

\section{Desarrollo}

La crisis financiera mundial dejará un aspecto positivo: los nuevos consumidores de productos financieros se preocupan cada vez más por entender dónde invierten sus ahorros y qué hará la entidad financiera con su dinero. Los escándalos, a pequeña y gran escala, han conseguido que el consumidor poco experto se preocupe, por fin, de tener una cultura financiera básica.

Perarnau (2003: 68) vaticinaba, pocos años antes, este cambio. Decía que todos los implicados en la formación financiera pretendían profundizar la cultura bursátil 
en España. Recogía que el número de quejas y consultas en el área del Supervisor de la CNMV (Comisión Nacional del Mercado de Valores) había crecido mucho, especialmente entre la población joven. De igual forma, reproducía las palabras de Petra Mateos, catedrática de la UNED (Universidad Nacional de Educación a Distancia): "Una mayor cultura financiera no debe ser exclusiva de los profesionales" (Perarnau, 2003: 68). Estas observaciones positivas no se extendían al grueso de la población española y la crisis serviría de varapalo y acicate para adquirir, por fin, a gran escala, esa cultura financiera.

Del mismo modo, la visión del consumidor hacia las entidades que le atienden, o que son susceptibles de hacerlo, ha cambiado. En España, tradicionalmente, los ciudadanos elegían banco o caja por proximidad al hogar o al lugar de trabajo, o por la confianza personal depositada en el empleado de la entidad financiera. La crisis también ha cambiado y ampliado estas razones y el consumidor ahora lee la letra pequeña de los contratos, desconfía hasta que logra una comprensión plena, y no se deja llevar simplemente por anuncios televisivos, cuñas radiofónicas o marquesinas callejeras.

Con ello, el logro y mantenimiento de una buena imagen se convierte en un fin más difícil y valioso de conseguir. Más todavía porque hablamos de ahorros y de confianza. Al respecto, debemos hacer la recomendación bibliográfica de Keita Karounga (2005: 20) por su carácter providencial y pertinente, recordando que se leyó tres años antes de la explosión de la crisis financiera en España. Precisamente, su autor dedicaba algunas líneas a hablar de la imagen como un nuevo bien importante, deseable y difícil de conseguir. Así es, una imagen positiva para las marcas las entidades financieras es clave pues consumidor no acudirá a ellas si no existe cierta confianza. Para las entidades, su imagen será un objetivo prioritario, igual que lo son los demás objetivos sobre rentabilidad. Así, los intangibles cobran el mismo valor que los balances tangibles. Para dar más ejemplos, el autor cita a Andrieu y Robinet (1993: 37) y dice cómo éstos también observaban que el sistema económico mundial estaba obligando a los empresarios a cuidar, cada vez más, la imagen.

En este sentido, las políticas de comunicación y marketing en banca juegan un papel importante y podrían ocupar una monografía completa, pues los estudios sobre marketing y finanzas sí disfrutan de una tradición académica importante. Por ello, reseñamos algunos de los trabajos más importantes de los últimos años: los manuales de los profesores Teruel Sierra (1995), Sánchez Guzmán (1997) o Embid, Zorrilla y Martín Dávila (1998); la obra de Sarro Álvarez sobre el marketing en cajas de ahorros (2001); el trabajo de Sundardas (2005) sobre nuevas estrategias en marketing financiero; y numerosos artículos de las revistas Harvard Business Review y MK: Marketing + ventas. Es un tema que siempre acapara interés y creemos que las estrategias de los bancos españoles, durante la crisis, generarán más manuales próximamente, que explicarán las campañas actuales que estamos viendo. Mientras eso ocurra, queremos plasmar algunas cuestiones fundamentales sobre marketing financiero, desarrolladas por los autores y que pueden servir de prospectiva para seguir investigando. 
Etxebarría y Barrutia (2002: 13) explicaban que tradicionalmente, se había empleado "el enfoque transaccional del marketing, vinculado a conceptos como las ventas aisladas de productos o la cuota de mercado". Sin embargo, esta corriente se estaba agotando y "este marketing transaccional ha sido oscurecido por el marketing relacional". Según los autores, la nueva forma de marketing enfatiza la fidelización del cliente y la cuota en la cartera del mismo: "No importa tanto cuantos productos se han vendido, sino en cuantos clientes se ha desarrollado el nivel de vinculación real o emocional con la empresa" (Etxebarría y Barrutia, 2002: 13). Unas líneas después, citan a Kotler (1999), que definía el marketing financiero como la ciencia y el arte de encontrar, conservar y desarrollar a los clientes valiosos. Asimismo, explican que la implantación de una estrategia de marketing relacional es muy recomendable en los sectores más competitivos (Etxebarría y Barrutia, 2002: 14). De este modo, consideramos que el marketing financiero debe alejarse del marketing tradicional para ver y entender al consumidor de otra forma y, de nuevo, ganarse su confianza.

En este contexto, se puede afirmar que las políticas de comunicación y marketing en las empresas en general se definen internamente bajo criterios intrínsecamente ligados a su propia cultura. En el caso de las empresas del sector financiero además están delimitadas por un marco regulador debido a la importancia de la estabilidad este sector dentro de la economía. Dentro de esa delimitación, se distinguen dos enfoques de la venta de los productos financieros: comercialización y asesoramiento (CNMV, 2010). La comercialización es considerada como una venta no asesorada. Mientras que el asesoramiento es una venta en la que existe una recomendación sobre unos instrumentos financieros concretos, ya sea de forma explícita o implícita. Además, es personalizada en el sentido de que es idónea para el inversor, basándose en sus circunstancias personales.

Así, la venta de productos financieros en la banca presenta unas características especiales con respecto al consumidor en cuanto a las comunicaciones que se realicen y el marketing que se haga de los mismos. Desde la primera regulación con la Ley 24/1988 del Mercado de Valores, esta delimitación venía determinada por las recomendaciones que la CNMV comunicaba al sector como buenas prácticas. La crisis financiera ha puesto al descubierto prácticas que no se ajustaron a esas recomendaciones en la venta de ciertos productos financieros entre la clientela minorista de las entidades financieras, como ha sido el caso de las participaciones preferentes en 2009. Esta problemática ha sido abordada en la Ley 9/2012, de 14 de noviembre, de reestructuración y resolución de entidades de crédito, que introdujo modificaciones en la Ley 24/1988, de 28 de julio, dando carácter imperativo a algunas de esas recomendaciones. De este modo, a partir de ese momento, la CNMV tiene la potestad de establecer que en la información y publicidad que se hagan de los productos financieros, se incluyan las advertencias que considere oportunas, las cuales han quedado descritas recientemente en la Circular 3/2013 de la CNMV (CNMV, 2013).

Tras haber explicado las formas de búsqueda de bibliografía científica y haber presentado un marco teórico y el estado de la cuestión general sobre los asuntos tratados, planteamos la banca islámica como posible referente para la banca tradicional. 
Así, vemos necesario reseñar concretamente aquellos autores que ya han estudiado ese enfoque. En primer lugar, destaca el estudio inicial de Sánchez (2006), que ya en la pasada década, auguraba los nuevos retos de la banca islámica. Varios años después, la misma autora hablaba del Islam en los medios de comunicación españoles (Sánchez y Padilla, 2013), otro asunto reseñable que bien daría lugar a una monografía completa e independiente. Asimismo, podemos recomendar la lectura de Lorca y Orozco (1999), que fueron los primeros en reseñar y compartir los elementos básicos de la banca islámica; seguidos de Iqbal (2006) y Abuamria (2006), que continuaron mostrando y analizando, en español, las principales características de este tipo de banca. Más adelante, Orozco (2008) regresó sobre la banca islámica, centrándose en nuevos mercados y nuevas alternativas. El mismo año, Anca (2008) hablaba de la banca islámica como alternativa a la incipiente crisis financiera y poco después, Nodal (2009) también se preguntaba si esta banca era una alternativa real a la banca tradicional.

Esta tradición continuó con otros autores que veían las posibilidades de la banca islámica y sobre todo, las características positivas que bien podrían imitar otras empresas financieras. Son los trabajos de García (2010), sobre las cajas de ahorro europeas; Imam y Kpodar (2010), acerca de cómo la banca islámica podría estimular el crecimiento de los países con población musulmana; Tornabell (2011), que se centró en las oportunidades en Cataluña; y Trullols (2011), que disertaba acerca de esperanzas y perspectivas que ofrecen las finanzas islámicas. Como decíamos en líneas anteriores, este trabajo bien puede ser una prospección académica para futuras investigaciones que continúen las líneas propuestas e intenten rellenar el vacío académico sobre el asunto. Sin embargo, antes de ello, y para fomentar la lectura y la investigación venideras, advertimos imprescindible dar los apuntes fundamentales que caracterizan la banca islámica. Atendiendo a los autores citados y a los conocimientos e investigaciones propios, pretendemos considerar aquí el Islam y la Banca Islámica como caso de estudio y posible e interesante alternativa a la banca tradicional.

La banca islámica se fundamenta en los principios islámicos, principalmente el de la equidad social que, entre otros, es la base de su filosofía respecto a la participación de resultado de las inversiones financieras. Sánchez González (2006: 61) cita en su trabajo que este principio de equidad se concreta en la concesión de financiación gratuita para los más necesitados y en la abolición de rendimientos fijos predeterminados, llamada "Riba", es decir, "usura", prohibida en el Corán (Sura 2). Esta prohibición supone la asociación directa de cualidades morales a un hecho económico, el cobro de intereses. Con ella se condena la usura. No se considera justo que el prestamista obtenga un beneficio sin hacer frente a ningún tipo de riesgo o esfuerzo productivo. En el Islam, toda ganancia debe estar sujeta a posibles pérdidas, a cierto componente de riesgo; o relacionada con el esfuerzo y el trabajo de los individuos. Por tanto, para legitimar todo enriquecimiento, se exige que se dé la creación de una contrapartida en forma de trabajo, participación en el riesgo de la actividad enriquecedora, responsabilidad sobre el producto comerciado y siempre garantizando que la participación de los diferentes miembros en la actividad sea equilibrada. 
También se prohíbe la existencia de "gharar", que se produce cuando se dan situaciones de información imperfecta o asimétrica en las transacciones económicas, bien en cuanto al precio o a las características del objeto en venta en el momento de firmar un contrato o realizar una transacción. Prohibiéndolo se intenta regular las transacciones económicas para que resulten justas, honestas y equitativas. Para ello, todas las partes en los contratos deben tener acceso a toda la información sobre los objetos del contrato: precio, costes, condiciones. Y se prohíbe, además, toda venta en la que el objeto no exista, esté fuera de control del vendedor, no tenga precio definido o se desconozca la fecha de su determinación. Por tanto, y de acuerdo con Sánchez González (2006), podemos decir que la banca islámica se aproxima al concepto de banca ética occidental, en el sentido de que parte del establecimiento de unos principios morales o éticos, en este caso los del Islam, como base de la organización y de todas sus actividades.

En este sentido, resulta oportuno señalar que la estructura de las instituciones bancarias islámicas es un reflejo de ese enfoque. Los bancos islámicos se crean a través de un contrato por el que varios socios se ponen de acuerdo para aportar unas cantidades determinadas de capital a la fundación del banco. En los pequeños, la toma de decisiones en la gestión del banco se crea por consenso entre los miembros fundadores. Cuando el número de inversores aumenta, las decisiones sobre cuestiones predeterminadas las toma un Consejo de representantes. El funcionamiento diario del banco lo lleva a cabo el Gabinete de Dirección, formado por un grupo de directores contratados, y otros departamentos vigilantes de esa ética como son, entre otros, el Comité de Supervisión Religioso, que introduce la justicia como principio regulador de la actividad bancaria; el departamento del "zakat", un impuesto islámico sobre la riqueza sobre la renta y ahorro que no participen en inversiones que, junto a la financiación de proyectos beneficiosos para la comunidad, constituye el principal rasgo social y redistributivo del banco; el departamento de Inversión, que realiza el seguimiento de las inversiones financiadas con los fondos del banco; y, por último, el departamento de Formación que se encarga de la preparación del personal en la teoría y práctica de la banca islámica además de la formación en temas de consultoría e inversiones (Sánchez González, 2006: 68).

Asimismo, los recursos del banco provienen de los fundadores, accionistas y clientes depositarios, que mantienen cuentas de inversión con participación en los beneficios del banco. Además, recibe ingresos de algunos servicios que ofrece a los clientes y de la parte de beneficios que le corresponden de las actividades económicas que haya ayudado a financiar. Asimismo, el uso de los recursos bancarios que se dirigen hacia la inversión del capital a través de los diferentes contratos islámicos también refleja ese nuevo enfoque. En estos tipos de contratos, el banco participa en el riesgo de la actividad financiada, tanto en sus beneficios, como en sus pérdidas. Dependiendo de la modalidad de contrato, también puede participar en la gestión de la actividad financiada o facilitar algún tipo de servicios relacionados con ella.

De este modo, existe y es posible llevar a cabo una actividad bancaria basada en una ética como la que establece el Islam a través de sus principios. Esto implica 
otro tipo de gestión financiera más transparente y consensuada. Los clientes de este tipo de entidades no tienen que ser necesariamente musulmanes. De hecho, en estos momentos de crisis económica, está constituyendo una oportunidad para que clientes de la banca tradicional y que no profesan el Islam, estén acudiendo a la banca islámica como alternativa. Igualmente, en los países musulmanes, cohabitan ambos tipos de banca.

\section{Conclusiones}

Descrita la situación y teniendo en cuenta que se necesitan cambios que provoquen, de nuevo, la confianza en los clientes, llegamos a la conclusión de que es posible y viable otra banca, otra comunicación, otra forma de entender los negocios bancarios y otras estrategias para acercarse al cliente del siglo XXI. Éste es mucho más exigente con los principios éticos, y más experimentado ante la posibilidad de elegir, en nuestro caso, qué tipo de banca prefiere para interactuar y depositar su confianza. Banca Ética, Microcréditos, Responsabilidad Social de la Empresa, Financiación 1to1, Banca Islámica y "crowdfunding" (financiación en masa), son algunas alternativas en plena expansión por los buenos resultados que arrojan, incluso en un contexto de crisis. En nuestro caso, creemos que es posible un cambio de modelo financiero.

En España, en los últimos años, la Junta Islámica y diferentes instituciones financieras han estudiado la posibilidad de crear un banco islámico. El primer paso es el lanzamiento de cuentas corrientes "halal", es decir, bajo la "garantía" de que el producto cumple con los principios éticos islámicos. Hasta ahora, los intentos han sido fallidos. Aunque todo parece indicar que en un futuro inmediato, se llegarán a acuerdos, sobre todo si tenemos en cuenta lo sucedido en países vecinos y socios europeos. Esperemos que nuestro próximo trabajo pueda analizar cómo se ha gestionado su inicio en nuestro país.

La banca islámica, como cualquier otro sistema bancario, debe contemplarse como un sistema en constante evolución. No sólo desde la perspectiva "glocal" -estrategias globales con adaptaciones locales-, sino también como alternativa intercultural de un sistema financiero dirigido a cualquier tipo de cliente, sea musulmán o no, que busque alternativas a lo que se ha constatado como sistema caduco. Cumple los requisitos religiosos del Islam pero también, creemos, amplía la cantidad de opciones disponibles a otros tipos de clientes, al ofrecer financiación de ventas y productos de bajo riesgo con productos basados en el reparto de riesgos y beneficios. Se trata de otra forma de realizar la función de intermediación financiera. Deberíamos empezar a cambiar ya nuestra forma de gestionarla. 


\section{Bibliografía}

ABUAMRIA, F. (2006). "La banca islámica en el mundo: principales rasgos y análisis de eficiencia". En Cuadernos de Información económica, ${ }^{\circ}$ 193. Disponible en: http://www.larioja.org/upload/documents/679368_CIE_N_193-2006. La_banca_islamica.pdf [10-9-2013].

ANDR̄IEU, S. y ROBINET, P. (1993). Les sondages pour l'entreprise. París: Éditions Eyrolles.

CHARLOT, B. (1994). "El enfoque cualitativo en las políticas educativas". En Perfiles educativos, $\mathrm{n}^{\circ}$ 63. Disponible en: http://www.oei.es/calidad2/enfoque. pdf [15-9-2013].

CNMV (2010). Guía sobre la prestación del servicio de asesoramiento en materia de inversión. Departamento de Supervisión ESI, CEA. 23 de diciembre

CNMV (2013). Circular 3/2013, de 12 de junio, de la Comisión Nacional del Mercado de Valores, sobre el desarrollo de determinadas obligaciones de información a los clientes a los que se les prestan servicios de inversión, en relación con la evaluación de la conveniencia e idoneidad de los instrumentos financieros. Boletín Oficial del Estado, miércoles 19 de junio de 2013. Sec. I. pág. 46150 - 46154.

DE ANCA, C. (2008). "Banca islámica y crisis del sistema financiero". En Afkar ideas: Revista trimestral para el diálogo entre el Magreb, España y Europa, $\mathrm{n}^{\mathrm{o}}$ 20. Disponible en: http://www.afkar-ideas.com/wp-content/uploads/files/3-20-15. pdf [15 de septiembre de 2013].

EMBID, P.; ZORRILLA, V. y MARTÍN, M. (1998). Marketing financiero. Madrid. McGraw-Hill Interamericana de España.

GARCÍA, A. (2010). Banca islámica: ¿una nueva oportunidad para las Cajas de Ahorros en Europa? En Ahorro, $\mathrm{n}^{\circ} 460$.

IMAM, P. y KPODAR, K. (2010). “Buena para el crecimiento? La expansión de la banca islámica puede estimular el crecimiento en los países con una amplia población musulmana". En Finanzas y desarrollo: publicación trimestral del Fondo Monetario Internacional y del Banco Mundial, Vol. 47, $\mathrm{n}^{\circ}$ 4. Disponible en: http://www.imf.org/external/pubs/ft/fandd/spa/2010/12/pdf/imam.pdf [15 de septiembre de 2013].

IQBAL, M. (2006). "Banca islámica: Teoría, práctica y evaluación”. En Papeles de economía española, $\mathrm{n}^{\circ}$ 110. Disponible en: http://europa.sim.ucm.es/compludoc/ AA?articuloId=539962 Consultado el [15 -9-2013].

KAROUNGA, K. (2005). La imagen corporativa de las entidades financieras. El caso del Banco Sabadell en Cataluña. Barcelona: Universidad Autónoma de Barcelona.

KOTLER, P (1999). El marketing según Kotler. Barcelona: Editorial Paidós.

LORCA, A. y OROZCO, O. (1999). La banca islámica sin intereses: elementos básicos. Madrid: Agencia Española de Cooperación Internacional.

NODAL, I. (2009). ¿Puede ser la banca islámica una alternativa real a la banca convencional?. En Revista de derecho del mercado de valores, $\mathrm{n}^{\circ}$ 4. La Ley. Madrid. 
OROZCO, O. (2008). "La banca islámica: nuevos mercados, ¿nuevas alternativas?". En Política exterior, Vol. 22, $\mathrm{n}^{\circ}$ 122. Disponible en: http://www.politicaexterior. com/archives/2357 Consultado el [10-9-2013].

PERARNAU, P. (2003). "La formación financiera en España hoy". En Bolsa de Madrid, $\mathrm{n}^{\circ}$ 122. Disponible en: http://www.bolsasymercados.es/esp/publicacion/ revista/2003/07/p68-73.pdf [15-9- 2013].

SÁNCHEZ, J.R. (1997). Marketing financiero: principios y estrategias. Madrid: Civitas.

SÁNCHEZ GONZÁLEZ, P. (2006). "La Banca Islámica: situación actual y retos". En Revista de la SEECI, $\mathrm{n}^{\circ}$ 13. Disponible en: http://www.seeci.net/Numeros/ Numero\%2013/PilarSan.pdf [15 de septiembre de 2013].

SÁNCHEZ GONZÁLEZ, P. y PADILLA, G. (2013). "La importancia del tratamiento de la religión en medios de comunicación. El caso del Islam en España". En Estudios sobre el Mensaje Periodístico, $\mathrm{n}^{\mathrm{o}}$ 19. Disponible en: http://revistas.ucm.es/ index.php/ESMP/article/view/42052/40033 [15 de septiembre de 2013].

SARRO, M.M. (2001). Marketing de las cajas de ahorros. Madrid: Ediciones Pirámide.

SOTELO, J. (2012). "Deporte y social media: el caso de la Primera División del fútbol español. En Historia y Comunicación Social, ${ }^{\circ}$ 17. Disponible en: http:// revistas.ucm.es/index.php/HICS/article/view/40607 [15 de septiembre de 2013].

SUNDARDAS, A. (2005). Marketing financiero: nuevas estrategias para el siglo $X X I$. Madrid: McGraw-Hill Interamericana de España.

TERUEL, M. (1995). Marketing financiero y de servicios de la oficina bancaria. Madrid: Universidad Pontificia Comillas.

TORNABELL, R. (2011). "La banca islàmica: una oportunitat de finançament per a Catalunya". En Via. Valors, idees, actituds: revista del Centre d'Estudis Jordi Pujol, $\mathrm{n}^{\mathrm{o}}$ 17. Disponible en: http://www.jordipujol.cat/files/articles/RTornabell. pdf [10 de septiembre de 2013].

TRULLOLS, C. (2011). "La banca islámica: esperanzas y perspectivas". En Economía exterior: estudios de la revista Política Exterior sobre la internacionalización de la economía española, $\mathrm{n}^{\circ}$ 57. Disponible en: http://www.politicaexterior.com/ archives/11355 [10 de septiembre de 2013].

\section{Las autoras}

$\mathrm{M}^{\mathrm{a}}$ del Carmen de la Orden de la Cruz. Actualmente, Profesora Titular (interina) de la Universidad Rey Juan Carlos de Madrid en el Departamento de Economía de la Empresa. Doctora en Ciencias Económicas y Empresariales y Máster en Gestión Financiera por la Universidad Complutense de Madrid. Desde 2007, Personal Docente e Investigador de la Universidad Rey Juan Carlos de Madrid en el Departa- 
mento de Economía de la Empresa, donde desarrolla actividades docentes, de gestión (lanzamiento y Directora del Máster Universitario en Asesoramiento y Planificación Financiera de la URJC entre 2009 y 2012) y de investigación. Anteriormente, entre 1990 y 1997, gestora de fondos de inversión en Gesmadrid, S.G.I.I.C. del grupo Caja Madrid y, entre 1998 y 2007, economista del Servicio de Estudios de Caja Madrid (actual Bankia).

Graciela Padilla Castillo. Profesora titular interina de la Facultad de Ciencias de la Información (Universidad Complutense de Madrid). Sus líneas de investigación abordan Teoría de la Información, Ficción televisiva, Ética audiovisual, Estudios de Género, Redes sociales y Redes profesionales, y Análisis Transaccional. Doctora en Ciencias de la Información, con Premio Extraordinario de Doctorado, ha completado su formación posdoctoral en la Universidad de California en Los Ángeles (UCLA). Es autora y coautora de más de sesenta capítulos de libro y artículos científicos. Miembro del Instituto de Investigaciones Feministas (Universidad Complutense de Madrid), colabora en la coordinación del Máster universitario en Comunicación Política y Empresarial (Universidad Camilo José Cela).

Pilar Sánchez González. Actualmente, Profesora del Departamento de Humanidades de ESIC. Realizando la Tesis Doctoral en la Universidad Rey Juan Carlos de Madrid. Licenciada en Sociología y en Ciencias Políticas. Master en Marketing Directo y Relacional por ESIC. Personal docente e investigador de ESIC desde 1993: profesora de Estructura Social, Ética Empresarial, Historia de las Instituciones Públicas y Marketing Directo y Relacional. Colabora periódicamente con universidades internacionales: ESAN en Lima, Perú; Fachhochschule en Dortmund, Alemania; y ESC, en Pau, Francia. Compagina la carrera académica con la Asesoría Profesional, y desde 1997, es directora de Gamón Pumareta Asesores en Marketing en Madrid. 